\title{
AMP-Activated Protein Kinase Alleviates Extracellular Matrix Accumulation in High Glucose-Induced Renal Fibroblasts through mTOR Signaling Pathway
}

\author{
Xia Luo Lingyan Deng Laxmi Pangeni Lamsal Wenjuan Xu Cheng Xiang \\ Liming Cheng
}

Department of Laboratory Medicine, Tongji Hospital, Tongji Medical College, Huazhong University of Science and Technology, Wuhan, China

\author{
Key Words \\ AMPK • Diabetic nephropathy $\bullet$ Extracellular matrix $\bullet$ mTOR
}

\begin{abstract}
Background/Aims: Extracellular matrix accumulation contributes significantly to the pathogenesis of diabetic nephropathy. Although AMP-activated protein kinase (AMPK) has been found to inhibit extracellular matrix synthesis by experiments in vivo and vitro, its role in alleviating the deposition of extracellular matrix in renal interstitial fibroblasts has not been well defined. Methods: Currently, we conducted this study to investigate the effects of AMPK on high glucose-induced extracellular matrix synthesis and involved intracellular signaling pathway by using western blot in the kidney fibroblast cell line (NRK-49f). Results: Collagen IV protein levels were significantly increased by high glucose in a time-dependent manner. This was associated with a decrease in Thr72 phosphorylation of AMPK and an increase in phosphorylation of mTOR on Ser2448. High glucose-induced extracellular matrix accumulation and mTOR activation were significantly inhibited by the co-treatment of rAAV-AMPK $\alpha 1^{312}$ (encoding constitutively active AMPK $\alpha 1$ ) whereas activated by r-AAV-AMPK $\alpha 1 D 157 A$ (encoding dominant negative AMPK $\alpha 1$ ). In cultured renal fibroblasts, overexpression of AMPK $\alpha 1 D 157 A$ upregulated mTOR signaling and matrix synthesis, which were ameliorated by co-treatment with the inhibitor of mTOR, rapamycin. Conclusion: Collectively, these findings indicate that AMPK exerts renoprotective effects by inhibiting the accumulation of extracellular matrix through mTOR signaling pathway.
\end{abstract}

Liming Cheng

KARGER 125
Department of Laboratory Medicine, Tongji Hospital, Tongji Medical College, Huazhong University of Science and Technology, Wuhan 430030 (China)

Tel. +86-27-836-50732, Fax +86-27-836-50732, E-Mail chengliming@tjh.tjmu.edu.cn 


\section{Introduction}

In 2012, 5\% of the total population suffered from diabetes mellitus (DM). As one of the most common complications of diabetes mellitus, diabetic nephropathy (DN) has become the leading cause of end-stage renal disease (ESRD) [1]. It is characterized by renal hypertrophy and excessive accumulation of extracellular matrix that results in interstitial fibrosis [2]. In the earlier stage of diabetic nephropathy, hyperglycemia can induce renal hypertrophy and stimulate the synthesis of the various matrix constituents which result in progressive thickening of the glomerular basement membranes (GBM) and tubular basement membranes (TBM). Most studies have focused on the increased deposition of extracellular matrix in the glomerular basement membrane and the mesangium, and their relation to disturbance in glomerular function but only a little is known about the pathogenesis and the significance of the thickened tubular basement membrane and the tubulointerstitial changes. Although many cell types are capable of generating ECM in the interstitium of kidneys, fibroblasts are commonly viewed as the principal matrix-producing cells that generate a large amount of ECM components including fibronectin, type I, type III and type IV collagens $[3,4]$. Thus, fibroblast activation in diabetes is important in the pathogenesis of renal fibrosis. Collagen is a major constituent of ECM. As a predominant component of glomerular basement membrane (GBM) and mesangial matrix, collagen IV is one potential biomarker in diabetic nephropathy [5]. Morita et al. reported that patients with Type I diabetes followed for 7 years demonstrated a significant association between urinary collagen IV and a decline in eGFR [6]. Similarly, Type II diabetic patients followed for 8 years also showed an inverse relationship between collagen IV and a decline in eGFR [7]. Therefore, inhibition of collagen IV accumulation in renal fibroblasts possibly provides a therapeutic target for interstitial fibrosis and then preserves renal function.

mTOR is a serine/threonine protein kinase that constitutes of two well known complexes (mTORC1 and mTORC2) [8]. It is emerged as an important modulator of several forms of renal disease, including diabetic nephropathy, polycystic kidney disease, podocyte dysfunction, and kidney fibrosis [9-20]. mTORC1 regulates phosphorylation of translation initiation regulators, p70S6 kinase and 4E-BP1 [21]. Additionally, mTOR-p70S6 kinase axis also has been found to control changes in phosphorylation of eukaryotic elongation factor 2 kinase (eEF2 kinase, Ser366), resulting in changes in the activity of elongation factors [22]. Therefore, mTORC1 constitutes a common regulatory factor for both initiation and elongation phases of translation. Rheb/mTORC1 signaling has been found to promote the activation of kidney fibroblasts that results in the development of interstitial fibrosis, and thus possibly providing a therapeutic target for the progressive renal disease [23].

AMPK is a ubiquitously expressed serine/threonine protein kinase that regulates energy metabolism, proliferation, inflammation, cytokine production and apoptosis. It is a newly identified regulator of renal hypertrophy in diabetes and has been shown to be inhibited in diabetic kidney [24]. Several researches revealed AMPK/mTOR signaling pathway might play a role in the extracellular matrix synthesis in mesangial cells or glomerular epithelial cells [25-27]. However, the role of AMPK signaling in renal interstitial fibrosis has not been well defined to date. AMPK activation by AICAR has been reported to negatively regulate mesangial-myofibroblast transdifferentiation, producing profibrotic effector cells that secrete and remodel ECM in vitro [28] and to ameliorate kidney fibrosis in a rat subtotal nephrectomy [29]. Another study also found AMPK could attenuate TGF- $\beta$, angiotensin II, aldosterone, high glucose and albumin induced epithelial-mesenchymal transition (EMT), which resulted in tubulointerstitial fibrosis [30]. Thus, AMPK activation could be a potential target for intervention of renal tubulointerstitial fibrosis in diabetic nephropathy. In fact, AICAR has recently been found to reduce tubulointerstitial fibrosis in UUO mice and inhibit TGF- $\beta$ induced kidney myofibroblast activation [31].

Although plenty of studies in a variety of cell types have indicated that AMPK negatively regulates protein synthesis, including matrix proteins, studies about the effects of AMPK in high glucose-induced dysfunction of the kidney fibroblast are still lacking. Therefore, 
Luo et al.: AMPK and Extracellular Matrix Accumulation in Renal Fibroblasts

subsequent experiments were conducted to examine the direct effects of AMPK on ECM synthesis in NRK-49f cells under high glucose condition and to highlight its potential role in prevention of renal interstitial fibrosis.

\section{Materials and Methods}

Recombinant adeno-associated virus (rAAV) preparation

The expression plasmids pcDNA3.1-AMPK $\alpha 1$ DN, encoding a dominant negative form of AMPK $\alpha 1$ (D157A) and pcDNA3.1-AMPK $\alpha 1$ CA, encoding a truncated, constitutively active form of AMPKa1 (1-312, T172D) were kindly provided by Dr. D. Carling (Imperial College School of Medicine, University of London, London, UK). An adenovirus expressing GFP was applied as a control. DNA fragments of AMPK $\alpha 1$ CA and DN were generated by RT-PCR using the primers CA-EcoRI-987-F (5'-GCCGAATTCATGGAGCAGAAGCTTATC-3'), CA-BglII-987-R (5'-CTGAGATCTTTAGTACAGGCAGCTGAG-3'), DN-EcoRI-1700-F (5'-GCCGAATTCATGGAGCAGAAGCTTATC-3'), and DN-BglII-1700-R (5'-CTG AGATCT TTACTGTGCAAGAATT-3'), respectively. Then, the DNA fragments were inserted into the EcoRI / BglII sites of the pSNAV vector (AGTC Gene Technology Co. Ltd., Beijing, China) to obtain the expression vectors designated pSNAV-AMPK $\alpha 1-C A$ and pSNAV-AMPK $\alpha 1-D N$. rAAVs containing AMPK $\alpha 1-C A$ and AMPKa1-DN were prepared according to the protocols. Briefly, BHK-21 cells (ATCC, CCL-10) were infected with pSNAVAMPK $\alpha 1$-CA and pSNAV-AMPK $\alpha 1$-DN plasmids by Lipofectamine ${ }^{\circledR} 2000$ (Invitrogen, Carlsbad, CA, USA) and selected by G418, respectively. The G418 resistant BHK-21 clones were infected with recombinant HSV1-rc/AUL2 helper viruses (AGTC Gene Technology Co. Ltd. Beijing, China) to produce rAAVs designated rAAV-AMPK $\alpha 1-C A$ and rAAV-AMPK $\alpha 1-D N$. After purification, titers of rAAVs were measured by dot-blot hybridization using a digoxin labeling and detection kit (Boehringer Mannheim, Mannheim, Germany). Concentration of rAAV-AMPK $\alpha 1$-CA is $3 \times 10^{12}$ (v.g.) $/ \mathrm{ml}$ and rAAV-AMPK $\alpha 1-D N$ is $1.14 \times 10^{12}$ (v.g.) $/ \mathrm{ml}$.

\section{Cell culture and treatment}

The kidney fibroblast cell line (NRK 49f) was from the American Type Culture Collection (Manasas, VA, USA). Cells were plated in 6-well plates in DMEM with $10 \%$ (v/v) FBS, 1\% (w/v) non essential amino acid, $1 \%(\mathrm{w} / \mathrm{v})$ L-glutamate and incubated in $5 \%(\mathrm{v} / \mathrm{v}) \mathrm{CO} 2$ atmosphere incubator at $37^{\circ} \mathrm{C}$. Before treatment, culture media was deprived of serum for 12 hours. After serum deprivation for 12 hours, cells were transfected with rAAV-AMPK $\alpha 1^{312}$, r-AAV-AMPK $\alpha 1$ D157A or rAAV-GFP for 24 hours. The transfected cells were then washed and cultured in fresh DMEM medium for an additional 12 hours prior to indicated treatment.

\section{Western blot}

All antibodies used in western blotting were purchased from Cell Signaling Technology (Beverly, MA, USA), except for $\beta$-actin (Santa Cruz Biotechnology, Santa Cruz, CA, USA), and collagen IV (Boster Biological Technology, Wuhan, China).

After treatment, NRK-49f cells were harvested and lysed in an ice-cold lysis buffer (Thermo Scientific, Rockford, IL, USA) containing $50 \mathrm{mM}$ Tris- $\mathrm{HCl}, 150 \mathrm{mM} \mathrm{NaCl}, 1 \%$ Triton X-100, 1\% sodium deoxycholate, $1 \%$ SDS, with protease and phosphatase inhibitors and a further 30 minutes shaking was performed on an orbital shaker at $4^{\circ} \mathrm{C}$. After centrifugation $\left(12,000 \times \mathrm{g}\right.$ for $15 \mathrm{~min}$ at $\left.4^{\circ} \mathrm{C}\right)$, the supernatants were transferred to new tubes and protein concentrations were determined by the Bradford Protein Assay Kit (Beyotime Institute of Biotechnology, Jiangsu, China). For Western blot, 15-20 $\mu$ g denaturated protein extract of each sample was separated on an $8 \%$ or $10 \%$ SDS-PAGE gel. After electrophoresis, proteins were transferred onto a polyvinylidene difluoride (PVDF) membrane (Bio-Rad, USA), $2 \mathrm{~h}$ for phosphor-ACC (Ser79), mTOR, phosphor-mTOR (Ser2448) and, $1 \mathrm{~h}$ for AMPK, phosphor-AMPK (Thr172), phospho-eEF2K (Ser366), phospho-p70S6k (Thr389) and $\beta$-actin, 25min for phospho-4EBP1 (Thr37/46) and collagen IV. Then membranes were blocked in 5\% w/v BSA in TBS for 3 hours at room temperature, and incubated with specific primary antibodies overnight $\left(4^{\circ} \mathrm{C}\right)$, respectively. After washing with TBST, the membranes were incubated with horseradish peroxidase (HRP)-conjugated secondary antibodies for $2 \mathrm{~h}$ at room temperature. The immunoreactivity was revealed by using an ECL kit (Thermo Scientific, Rockford, IL, USA). 
Fig. 1. Time-dependent effects of high glucose on matrix protein expression in the kidney fibroblasts. NRK-49f cells were quiesced in serum-free medium for $12 \mathrm{~h}$ followed by treatment with $30 \mathrm{mM}$ D-glucose for different periods of time. Control cells were cultured in normal medium with $5 \mathrm{mM}$ glucose. Equal amount of cell lysates was separated by SDSPAGE followed by Western blot analysis for Col IV and the results were normalized to the cells in the media with $5 \mathrm{mM}$ glucose. A representative blot and composite data from three independent experiments are shown in this graph. *, $p<0.05$, for different time periods vs. $0 \mathrm{~h}$.

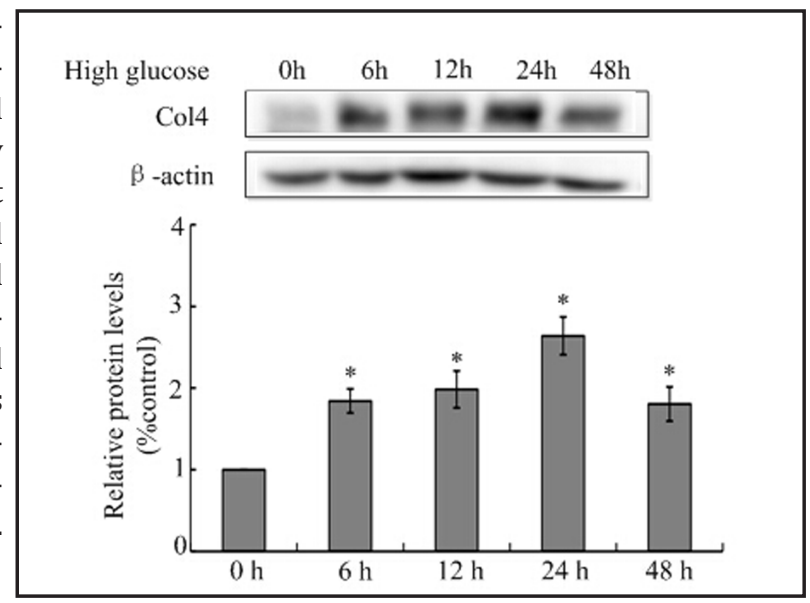

\section{Statistical Analysis}

Each experiment was repeated at least three times. Differences among the groups were assessed by ANOVA, after which unpaired t test was applied for comparison of two groups. All results were analyzed by SPSS 20.0 software and $P<0.05$ was considered statistically significant.

\section{Results}

High glucose-induced extracellular matrix synthesis in kidney fibroblasts

Kidney fibroblasts were exposed to a high glucose environment periodically in diabetic nephropathy. $30 \mathrm{mM}$ glucose was applied to mimic hyperglycemia of diabetes mellitus in this study. After $12 \mathrm{~h}$ of incubation in serum-deprived medium, NRK-49f cells were cultured with high glucose for $0,6,12,24,48 \mathrm{~h}$. Collagen IV, a major component of the matrix, was examined by western blot. The expression of collagen IV protein in high glucose group started to increase at $6 \mathrm{~h}$ and the effect peaked at 24h (Fig. 1). Results indicated that high glucose induced high levels of collagen IV expression in renal fibroblasts in a time dependent manner.

AMPK was involved in high glucose-induced extracellular matrix synthesis in NRK-49f cells As one of the downstream targets of AMPK, acetyl-CoA carboxylase (ACC) plays a key role in regulation of mammalian fatty acid synthesis. Phosphorylation at Thr-172 of AMPK $\alpha 1$ and Ser79 of ACC are now almost universally considered as biomarkers to evaluate AMPK activation [32]. In the present study, we examined whether high glucose could inhibit AMPK activity in serum-deprived kidney fibroblasts (NRK-49F cells) in vitro. As illustrated in Fig. $2 \mathrm{~A}$, high glucose addition rapidly reduced the level of AMPK phosphorylation in a timedependent manner. The reduction in Thr172 phosphorylation of AMPK corresponded to an obvious decrease in ACC phosphorylation (Ser-79) (Fig. 2B).

To determine whether or not the activity of AMPK $\alpha$ was associated with the change in matrix protein synthesis, a constitutively active truncated AMPK $\alpha 1^{312}$ (CA) or catalytically inactive mutant AMPK $\alpha 1 \mathrm{D} 157 \mathrm{~A}$ (DN) by rAAV-mediated transfection was applied to activate or inhibit AMPK. AMPK phosphorylation level was increased in AMPK $\alpha 1^{312}$ over-expressing cells while overexpression of AMPK $\alpha 1 D 157$ A significantly impaired AMPK activity as shown by the phosphorylation levels of AMPK and ACC (Fig. 2C). Moreover, treatment with rAAVAMPK $\alpha 1^{312}$ caused obvious inhibition of high glucose-induced collagen IV expression while rAAV-AMPK $\alpha 1 D 157 \mathrm{~A}$ upregulated that level compared with the group without treatment. Taking together, the changes of extracellular matrix synthesis were altered by the activity of AMPK in high glucose environment. Thus, high glucose inhibits AMPK phosphorylation to regulate extracellular matrix protein expression in the NRK-49f cells. 


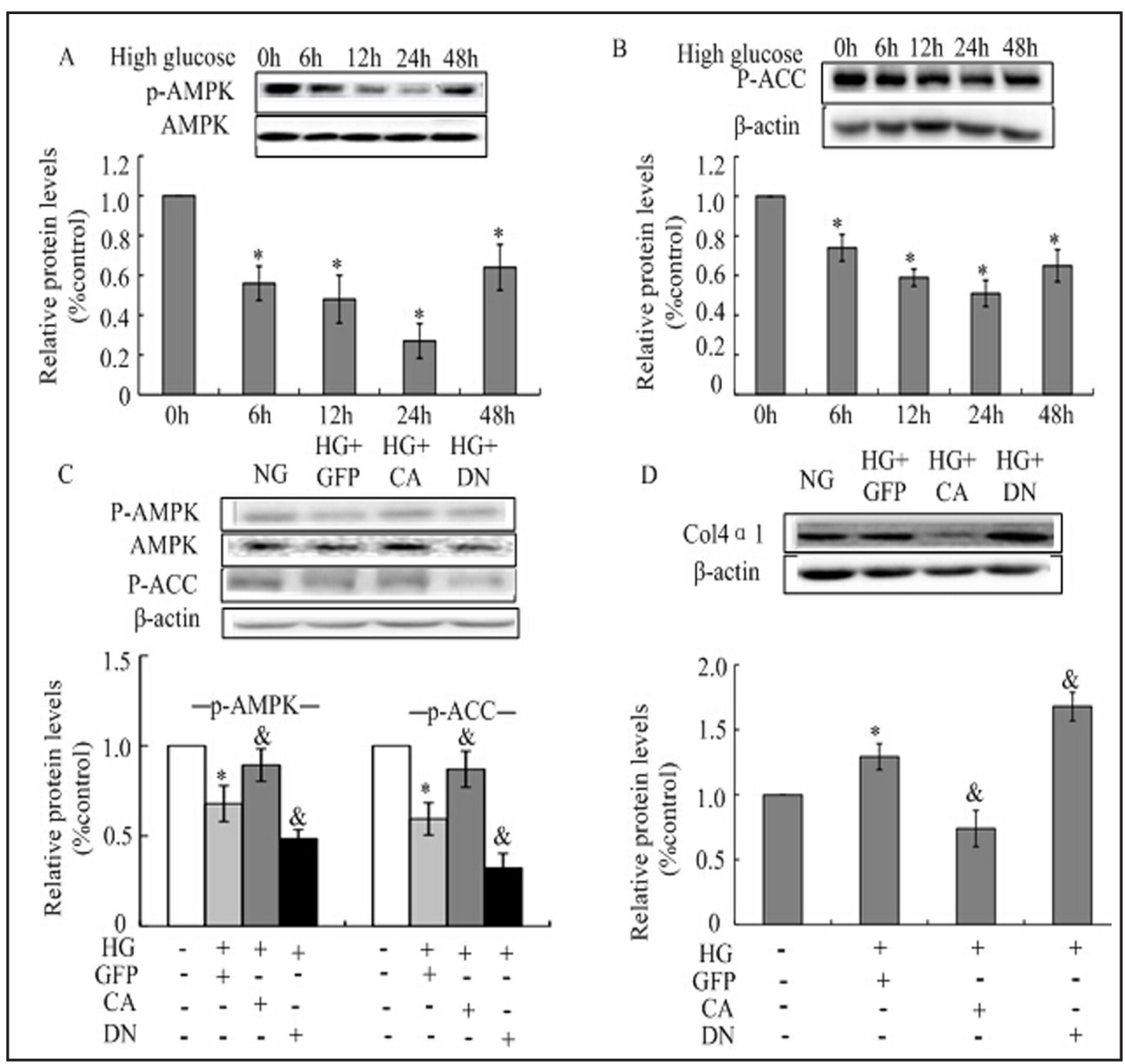

Fig. 2. AMPK mediated high glucose-induced matrix Col IV increment in the kidney fibroblasts. Quiescent NRK-49f cells were cultured in $30 \mathrm{mM}$ D-glucose for different periods of time for studies on effects of high glucose on AMPK activity. Equal amount of cell lysate was separated by SDS-PAGE and immunoblotted with antibodies against total and phosphorylated AMPK (Thr72) (A), and phosphorylated ACC (Ser79) (B). Quiescent cells were then transfected for $24 \mathrm{~h}$ with recombinant adeno-associated virus vectors expressing AMPK $\alpha 1^{312}$ or AMPK $\alpha 1 D 157 A$ or GFP. The transfected cells were washed and cultured in fresh DMEM medium for $12 \mathrm{~h}$, after which they were treated with $30 \mathrm{mM}$ high glucose for an additional $24 \mathrm{~h}$. Total and phosphorylated AMPK (Thr72), and phosphorylated ACC (Ser79) (C), Col IV in transfected cell lysates (D) were also measured, and the results were normalized to the cells in the media with $5 \mathrm{mM}$ glucose. Representative blots and composite graph from three independent experiments are shown. ${ }^{*}, p<0.05$ vs. cells without treatment, $\&, p<0.05$ vs. cells in medium with high glucose.

\section{Activation of mTOR signaling pathway induced by high glucose in NRK-49f cells}

As presented above, preincubation of cells with high glucose could induce collagen IV expression and inhibit AMPK activity in a time dependent manner. Then, we investigated the changes of effectors in mTOR signaling pathways. In a time course experiment in NRK$49 \mathrm{f}$ cells, the phosphorylation level of mTOR (Ser2448) was increased in response to high glucose in a time dependent manner, with maximal induction at 24h. Additionally, high glucose resulted in increased amount of phosphorylated p70S6 kinase, 4EBP1, and eEF2 kinase, which were key regulators of matrix protein synthesis for up to $48 \mathrm{~h} \mathrm{(Fig.} \mathrm{3).}$ 


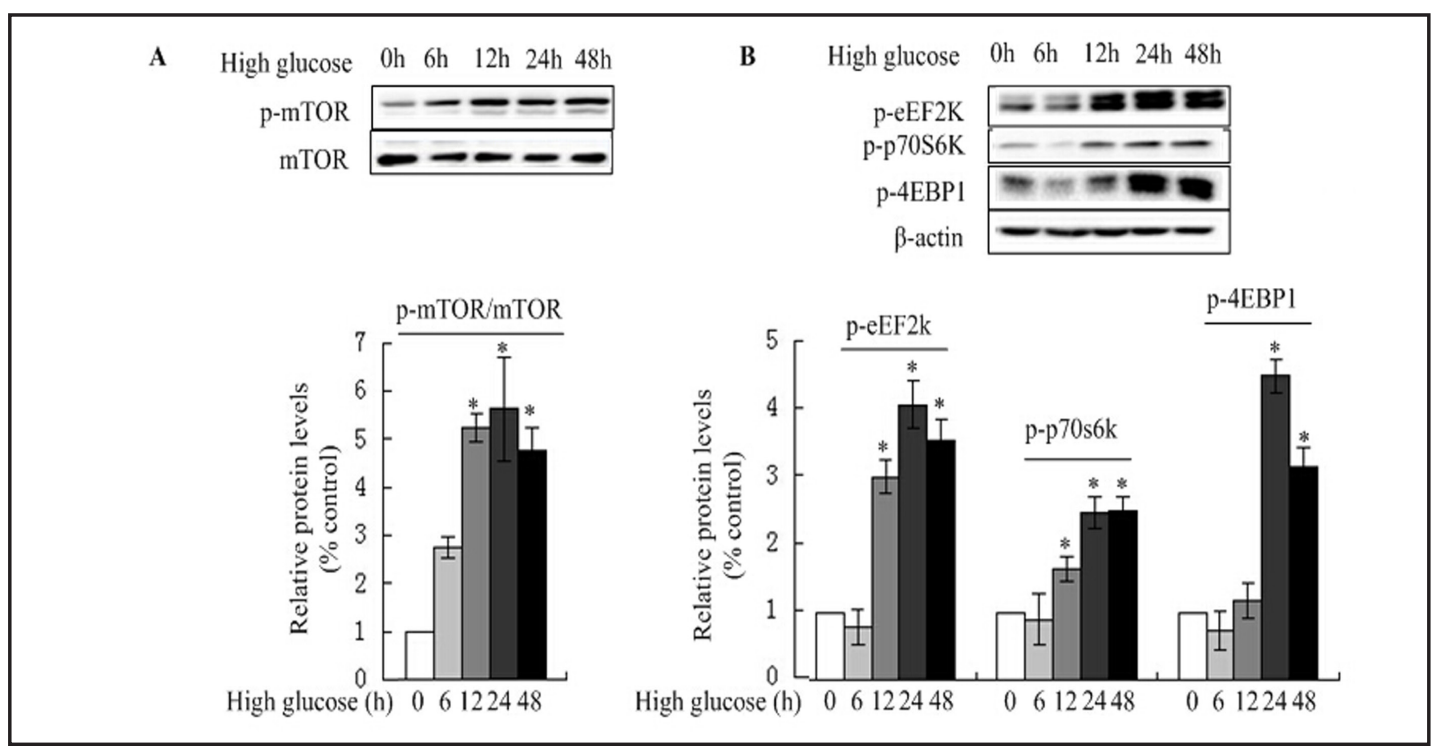

Fig. 3. Time-dependent effects of high glucose on mTOR signaling pathway in the kidney fibroblasts. Equal amount of cell lysates was separated by SDS-PAGE and immunoblotted with specific antibodies. Immunoblotting was done with antibodies against total and phosphorylated mTOR (Ser2448) (A), phosphorylated p70S6k (Thr389), 4E-BP1 (Thr37/46) and eEF2k (Ser366) (B). The results were normalized to the cells in the media with $5 \mathrm{mM}$ glucose. Representative blots and composite graph from three independent experiments are shown. ${ }^{*}, p<0.05$, for different time periods vs. $0 \mathrm{~h}$.

Fig. 4. AMPK mediated high glucose-stimulated mTOR signal activation in the kidney fibroblasts. Quiescent cells were transfected for $24 \mathrm{~h}$ with recombinant adeno-associated virus vectors expressing $A M P K \alpha 1^{312}$ or AMPK $\alpha 1 D 157 A$ or GFP. The transfected cells were then washed and cultured in fresh DMEM medium for $12 \mathrm{~h}$, after which they were treated with $30 \mathrm{mM}$ glucose for an additional $24 \mathrm{~h}$. Total and phosphorylated mTOR (Ser2448), phosphorylated p70S6k (Thr389), 4E-BP1 (Thr37/46) and eEF2k (Ser366) were measured. The results were normalized to cells in the media with $5 \mathrm{mM}$ glucose. Representative blots and composite graph from three independent experiments are shown. *, $\mathrm{p}<0.05$ vs. cells without treatment, $\&, p<0.05$ vs. cells in medium with high glucose.

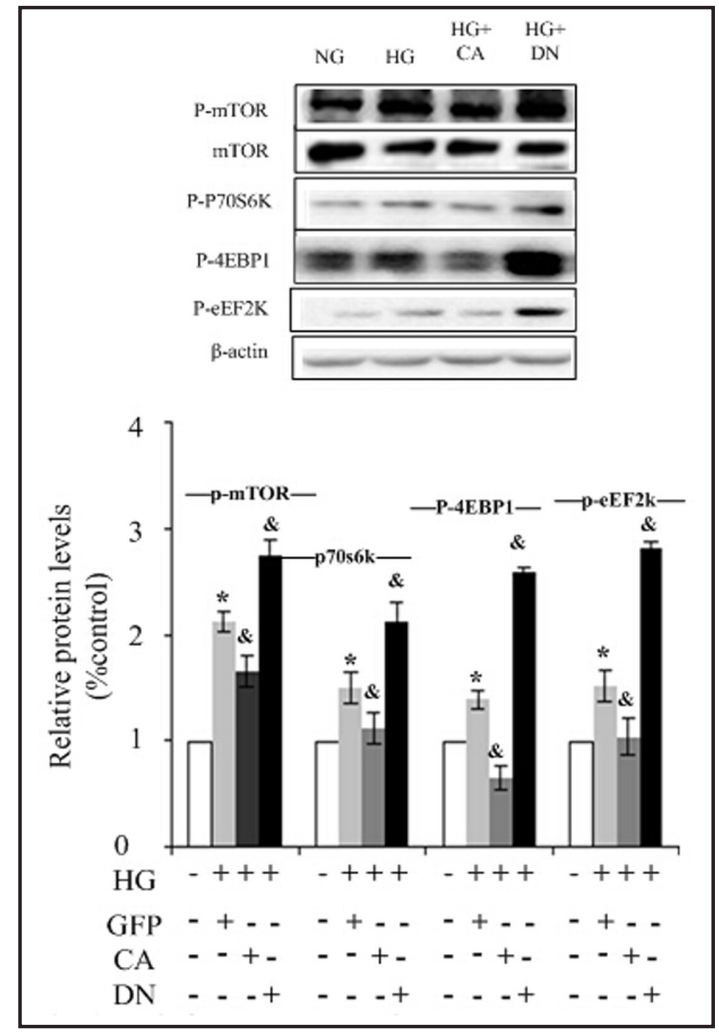

AMPK negatively regulated $m$ TOR pathway in response to high glucose

To examine whether or not inhibition of AMPK in response to high glucose might result in changes in mTOR activity and thereby alter phosphorylation of p70s6 kinase, 4EBP1 and eEF2 kinase, cells were transfected with recombinant adeno-associated virus vectors 


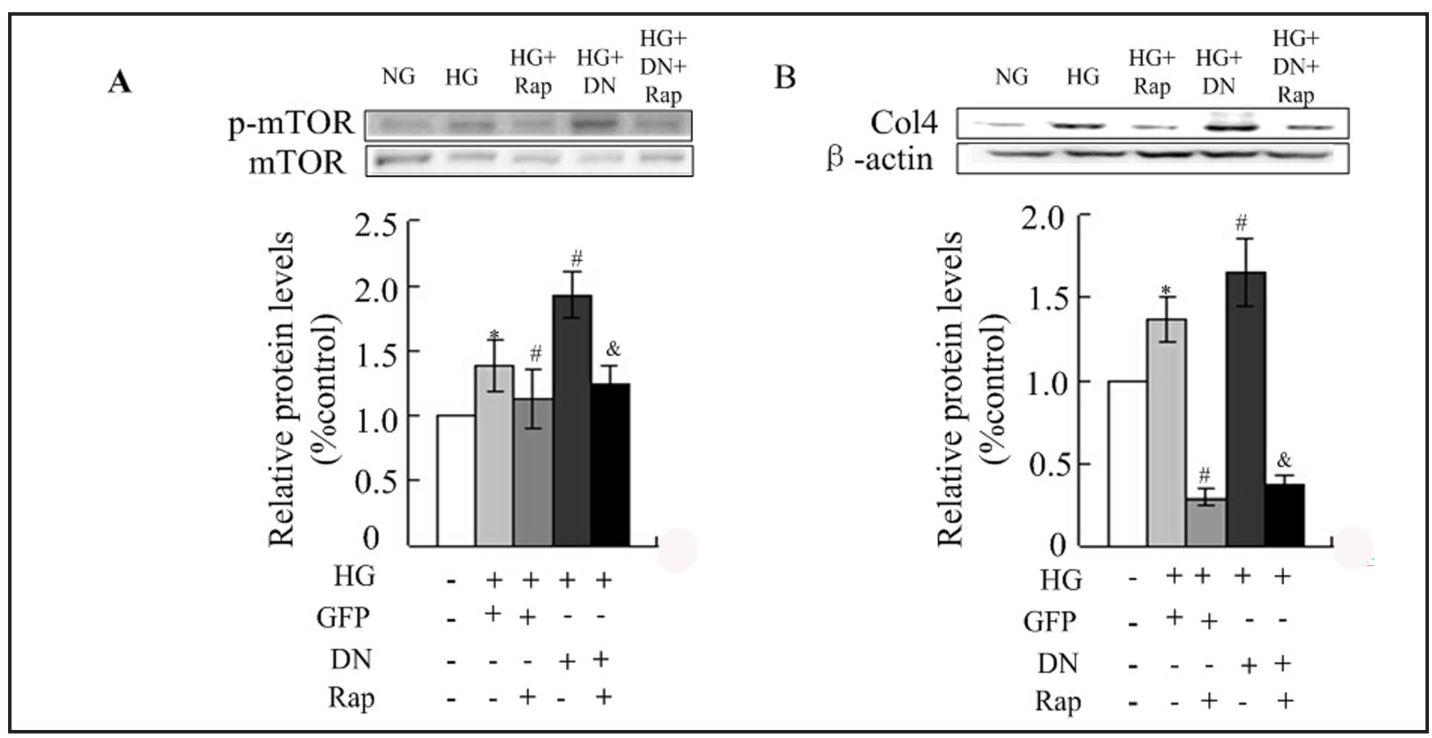

Fig. 5. AMPK inhibited matrix expression induced by high glucose through mTOR pathway. Quiescent cells were transfected for $24 \mathrm{~h}$ with recombinant adeno-associated virus vectors expressing AMPK $\alpha 1 \mathrm{D} 157 \mathrm{~A}$ or GFP. The transfected cells were then washed and cultured in fresh DMEM medium for $12 \mathrm{~h}$, after which they were cultured in medium with $30 \mathrm{mM}$ glucose with or without $10 \mathrm{nM}$ rapamycin for an additional $24 \mathrm{~h}$. Total and phosphorylated mTOR (Ser2448) (A), Col IV in cell lysates (B) were examined. The results were normalized to the cells in the media with $5 \mathrm{mM}$ glucose. Representative blots and composite graph from three independent experiments are shown. ${ }^{*}, p<0.05$ vs. cells without treatment, $\#, p<0.05$ vs. cells in medium with high glucose, $\&, p<0.05$ vs. cells transfected with AMPK $\alpha 1 \mathrm{D} 157 \mathrm{~A}$.

encoding AMPK $\alpha 1^{312}$ or AMPK $\alpha 1 D 157$ A. Protein immunoblot analysis was conducted using antibodies specific for phosphorylated mTOR (Ser2448), p70S6k (Thr389), 4EBP1 (Thr37/46) and eEF2k (Ser366). As shown in Fig. 4, constitutively activated AMPK attenuated drastically the amount of phosphorylated mTOR, p70s6 kinase, 4EBP1 and eEF2 kinase compared with that of only high glucose exposure and this variation was reversed by rAAV-AMPK $\alpha 1 D 157$ A treatment.

AMPK attenuated high glucose-induced matrix synthesis through mTOR signaling

Previous studies demonstrated that AMPK suppressed protein synthesis through down-regulated mTOR signaling in skeletal muscles [21]. In consideration of the confirmed inhibitory effects of AMPK on high glucose-induced ECM synthesis and mTOR signaling in this study, we therefore question whether AMPK suppresses high glucose-induced ECM synthesis via mTOR cascades. We examined the effect of rapamycin, an inhibitor of mTOR, on mTOR activity. As expected, rapamycin inhibited mTOR activity in a dose-dependent manner and rapamycin at $10 \mathrm{nM}$ most significantly reduced the activity of mTOR (data not shown). Based on the results from the dose-dependent experiments, rapamycin was used at $10 \mathrm{nM}$ in the following experiments. To examine how AMPK activation attenuated high glucoseinduced ECM synthesis through mTOR signaling pathway, we first checked expression level of mTOR phosphorylation in response to dominant negative mutant of AMPK (AMPKDN) or/and rapamycin, respectively. As shown in Fig. 5A, rapamycin obviously inhibited mTOR activity and inhibition of AMPK by rAAV-AMPK-DN increased the phosphorylation of mTOR, which was clearly abrogated by co-treatment of rapamycin. Next, we examined the expression of collagen IV after the indicated treatment. Similarly, rapamycin significantly alleviated the effect of r-AAV-AMPK $\alpha 1$ D157A on collagen IV synthesis (Fig. 5B), indicating that AMPK could inhibit matrix synthesis though mTOR signaling. 


\section{Discussion}

Extracellular matrix accumulation (ECM) is an important factor in the progression of renal disease. When exposed to high glucose, mesangial cells expressed a high level of extracellular matrix proteins, leading to the progression of renal disease and eventually glomerulosclerosis in human and animals with diabetes [33]. In the current study, we demonstrated AMPK could obviously inhibit high glucose-induced collagen IV synthesis in rat kidney interstitial fibroblasts. Moreover, AMPK inhibited the activation of mTOR and p70S6 kinase, restoring the changes in initiation and elongation phases of mRNA translation induced by high glucose. After that, we explored the underlying mechanism and demonstrated that AMPK blocked high glucose-induced ECM synthesis through downregulating mTOR signaling. Taken together, it is implied that AMPK can potentially alleviate the renal dysfunction related with ECM accumulation in NRK-49f cells.

AMPK is composed of the catalytic subunit $(\alpha)$, scaffolding subunit $(\beta)$ and regulatory subunit $(\gamma)[34,35]$. It is abundantly expressed in the kidney where it is involved in a variety of pathophysiological processes, including acute renal ischemia, renal cystogenesis, diabetic or gentamycin-induced nephropathy [24, 36-38]. It serves as a part of the AMPKTSC signaling pathway and inhibits Rheb/mTORC1 and protein synthesis [39]. Our results showed that high glucose could inhibit AMPK activation with a significant decrease of phosphorylated ACC time dependently in NRK-49f cells. Accompanied by the decreased AMPK activity, high glucose also resulted in a significant increase of collagen IV synthesis. Moreover, constitutively activated AMPK effectively ameliorated collagen IV expression which was increased by inhibition of AMPK by rAAV-AMPK-DN. Such results suggest that ECM accumulation induced by high glucose is related to the inhibition of AMPK activity.

Most of the beneficial actions of AMPK are accounted for by the inhibition of the mammalian target of rapamycin (mTOR). Inhibition of LKB1/AMPK signaling pathway and subsequent activation of mTOR/p70S6k signaling have been proposed to promote hypertrophic growth [40]. mTOR plays an important role in stimulating protein translation and phosphorylates 4E-BP1 and p70S6 kinase, the translation initiation regulators [21]. In addition to initiation phase, mTOR-p70S6 kinase axis controls some steps in the elongation phase of translation such as phosphorylation of eEF2 kinase [22]. Here, we examined the effect of high glucose on the mTOR signaling activation and found that high glucose significantly increased the phosphorylation of mTOR, p70s6 kinase, 4EBP1 and eEF2 kinase in NRK-49f cells. mTOR signaling activation was repressed in AMPK-CA-overexpressed cells while that was increased by AMPK-DN treatment. Together the changes in 4E-BP1, p70s6 kinase and eEF2 kinase phosphorylation suggest that translation-promoting mTOR pathway is negatively regulated by AMPK.

The question of whether the decreased AMPK activity induced by high glucose mediates its effect on mTOR/p70S6K signaling and consequently, matrix protein synthesis is unresolved. As shown in Fig. 5, our results revealed that overexpression of AMPK-DN could upregulate the activity of mTOR and increase the expression of collagen IV. However, rapamycin suppressed the effects of AMPK-DN on mTOR activation and matrix synthesis, confirming that the inhibitory effects of AMPK on high glucose-induced ECM accumulation are mediated by mTOR signaling.

In conclusion, AMPK regulated ECM accumulation by inhibiting mTOR signaling pathway, consequently alleviated the progression of renal fibrosis. Further testing is warranted to determine the anti-fibrotic effect of AMPK in vivo.

\section{Acknowledgments}

This work was supported by grants from National Natural Science Foundation of China (81000331). 
Luo et al.: AMPK and Extracellular Matrix Accumulation in Renal Fibroblasts

\section{Disclosure Statement}

The authors declare that they have no conflicts of interest.

\section{References}

1 Papale M, Di Paolo S, Magistroni R, Lamacchia O, Di Palma AM, De Mattia A, Rocchetti MT, Furci L, Pasquali S, De Cosmo S, Cignarelli M, Gesualdo L: Urine proteome analysis may allow noninvasive differential diagnosis of diabetic nephropathy. Diabetes care 2010;33:2409-2415.

-2 Chuang LY, Guh JY: Extracellular signals and intracellular pathways in diabetic nephropathy. Nephrology 2001;6:165-172.

- Liu Y: Cellular and molecular mechanisms of renal fibrosis. Nat Rev Nephrol 2011;7:684-696.

4 Shiohira S, Yoshida T, Sugiura H, Nishida M, Nitta K, Tsuchiya K: Sphingosine-1-phosphate acts as a key molecule in the direct mediation of renal fibrosis. Physiol Rep 2013;1:e00172.

5 Jim B, Santos J, Spath F, Cijiang He J: Biomarkers of diabetic nephropathy, the present and the future. Curr Diabetes Rev 2012;8:317-328.

6 Morita M, Uchigata Y, Hanai K, Ogawa Y, Iwamoto Y: Association of urinary type iv collagen with gfr decline in young patients with type 1 diabetes. Am J Kidney Dis 2011;58:915-920.

7 Araki S, Haneda M, Koya D, Isshiki K, Kume S, Sugimoto T, Kawai H, Nishio Y, Kashiwagi A, Uzu T, Maegawa $\mathrm{H}$ : Association between urinary type iv collagen level and deterioration of renal function in type 2 diabetic patients without overt proteinuria. Diabetes care 2010;33:1805-1810. Cornu M, Albert V, Hall MN: Mtor in aging, metabolism, and cancer. Curr Opin Genet Dev 2013;23:53-62. Dazert E, Hall MN: Mtor signaling in disease. Curr Opin Cell Biol 2011;23:744-755.

Lloberas N, Cruzado JM, Franquesa M, Herrero-Fresneda I, Torras J, Alperovich G, Rama I, Vidal A, Grinyo JM: Mammalian target of rapamycin pathway blockade slows progression of diabetic kidney disease in rats. J Am Soc Nephrol 2006;17:1395-1404.

11 Novalic Z, van der Wal AM, Leonhard WN, Koehl G, Breuning MH, Geissler EK, de Heer E, Peters DJ: Dose-dependent effects of sirolimus on mtor signaling and polycystic kidney disease. J Am Soc Nephrol 2012;23:842-853.

12 Perico N, Antiga L, Caroli A, Ruggenenti P, Fasolini G, Cafaro M, Ondei P, Rubis N, Diadei O, Gherardi G, Prandini S, Panozo A, Bravo RF, Carminati S, De Leon FR, Gaspari F, Cortinovis M, Motterlini N, EneIordache B, Remuzzi A, Remuzzi G: Sirolimus therapy to halt the progression of adpkd. J Am Soc Nephrol 2010;21:1031-1040.

13 Qin S, Taglienti M, Nauli SM, Contrino L, Takakura A, Zhou J, Kreidberg JA: Failure to ubiquitinate c-met leads to hyperactivation of mtor signaling in a mouse model of autosomal dominant polycystic kidney disease. J Clin Invest 2010;120:3617-3628.

14 Weimbs T: Polycystic kidney disease and renal injury repair: Common pathways, fluid flow, and the function of polycystin-1. Am J Physiol Renal Physiol 2007;293:F1423-1432.

15 Habib SL, Yadav M, Tizani S, Bhandari B, Valente AJ: Tuberin inhibits production of the matrix protein fibronectin in diabetes. J Am Soc Nephrol 2012;23:1652-1662.

16 Shegogue D, Trojanowska M: Mammalian target of rapamycin positively regulates collagen type i production via a phosphatidylinositol 3-kinase-independent pathway. J Biol Chem 2004;279:23166-23175.

-17 Sola-Villa D, Camacho M, Sola R, Soler M, Diaz JM, Vila L: Il-1beta induces vegf, independently of pge2 induction, mainly through the pi3-k/mtor pathway in renal mesangial cells. Kidney Int 2006;70:19351941.

18 Vollenbroker B, George B, Wolfgart M, Saleem MA, Pavenstadt H, Weide T: Mtor regulates expression of slit diaphragm proteins and cytoskeleton structure in podocytes. Am J Physiol Renal Physiol 2009;296:F418426.

19 Wang S, Wilkes MC, Leof EB, Hirschberg R: Noncanonical tgf-beta pathways, mtorc1 and abl, in renal interstitial fibrogenesis. Am J Physiol Renal Physiol 2010;298:F142-149.

20 Chen JK, Chen J, Neilson EG, Harris RC: Role of mammalian target of rapamycin signaling in compensatory renal hypertrophy. J Am Soc Nephrol 2005;16:1384-1391. 
Luo et al.: AMPK and Extracellular Matrix Accumulation in Renal Fibroblasts

21 Bolster DR, Crozier SJ, Kimball SR, Jefferson LS: Amp-activated protein kinase suppresses protein synthesis in rat skeletal muscle through down-regulated mammalian target of rapamycin (mtor) signaling. J Biol Chem 2002;277:23977-23980.

-22 Sataranatarajan K, Mariappan MM, Lee MJ, Feliers D, Choudhury GG, Barnes JL, Kasinath BS: Regulation of elongation phase of mrna translation in diabetic nephropathy: Amelioration by rapamycin. Am J Pathol 2007;171:1733-1742.

23 Jiang L, Xu L, Mao J, Li J, Fang L, Zhou Y, Liu W, He W, Zhao AZ, Yang J, Dai C: Rheb/mtorc1 signaling promotes kidney fibroblast activation and fibrosis. J Am Soc Nephrol 2013;24:1114-1126.

-24 Lee MJ, Feliers D, Mariappan MM, Sataranatarajan K, Mahimainathan L, Musi N, Foretz M, Viollet B, Weinberg JM, Choudhury GG, Kasinath BS: A role for amp-activated protein kinase in diabetes-induced renal hypertrophy. Am J Physiol Renal Physiol 2007;292:F617-627.

25 Lee HJ, Mariappan MM, Feliers D, Cavaglieri RC, Sataranatarajan K, Abboud HE, Choudhury GG, Kasinath BS: Hydrogen sulfide inhibits high glucose-induced matrix protein synthesis by activating amp-activated protein kinase in renal epithelial cells. J Biol Chem 2012;287:4451-4461.

26 Lee MJ, Feliers D, Sataranatarajan K, Mariappan MM, Li M, Barnes JL, Choudhury GG, Kasinath BS: Resveratrol ameliorates high glucose-induced protein synthesis in glomerular epithelial cells. Cell Signal 2010;22:65-70.

27 Gorin Y, Block K, Hernandez J, Bhandari B, Wagner B, Barnes JL, Abboud HE: Nox4 nad(p)h oxidase mediates hypertrophy and fibronectin expression in the diabetic kidney. J Biol Chem 2005;280:3961639626.

28 Mishra R, Cool BL, Laderoute KR, Foretz M, Viollet B, Simonson MS: Amp-activated protein kinase inhibits transforming growth factor-beta-induced smad3-dependent transcription and myofibroblast transdifferentiation. J Biol Chem 2008;283:10461-10469.

29 Satriano J, Sharma K, Blantz RC, Deng A: Induction of ampk activity corrects early pathophysiological alterations in the subtotal nephrectomy model of chronic kidney disease. Am J Physiol Renal Physiol 2013;305:F727-733.

-30 Lee JH, Kim JH, Kim JS, Chang JW, Kim SB, Park JS, Lee SK: Amp-activated protein kinase inhibits tgf-beta-, angiotensin ii-, aldosterone-, high glucose-, and albumin-induced epithelial-mesenchymal transition. Am J Physiol Renal Physiol 2013;304:F686-697.

- 31 Chen KH, Hsu HH, Lee CC, Yen TH, Ko YC, Yang CW, Hung CC: The ampk agonist aicar inhibits tgf-beta1 induced activation of kidney myofibroblasts. PLoS One 2014;9:e106554.

- 32 Hardie DG: Ampk: A target for drugs and natural products with effects on both diabetes and cancer. Diabetes 2013;62:2164-2172.

-33 Mason RM, Wahab NA: Extracellular matrix metabolism in diabetic nephropathy. J Am Soc Nephrol 2003;14:1358-1373.

34 Hardie DG: Amp-activated/snf1 protein kinases: Conserved guardians of cellular energy. Nat Rev Mol Cell Biol 2007;8:774-785.

-35 Hardie DG, Ross FA, Hawley SA: Ampk: A nutrient and energy sensor that maintains energy homeostasis. Nat Rev Mol Cell Biol 2012;13:251-262.

-36 Morales AI, Detaille D, Prieto M, Puente A, Briones E, Arevalo M, Leverve X, Lopez-Novoa JM, El-Mir MY: Metformin prevents experimental gentamicin-induced nephropathy by a mitochondria-dependent pathway. Kidney Int 2010;77:861-869.

-37 Mount PF, Hill RE, Fraser SA, Levidiotis V, Katsis F, Kemp BE, Power DA: Acute renal ischemia rapidly activates the energy sensor ampk but does not increase phosphorylation of enos-ser1177. Am J Physiol Renal Physiol 2005;289:F1103-1115.

-38 Takiar V, Nishio S, Seo-Mayer P, King JD, Jr., Li H, Zhang L, Karihaloo A, Hallows KR, Somlo S, Caplan MJ: Activating amp-activated protein kinase (ampk) slows renal cystogenesis. Proc Natl Acad Sci U S A 2011;108:2462-2467.

-39 Mariappan MM: Signaling mechanisms in the regulation of renal matrix metabolism in diabetes. Exp Diabetes Res 2012;2012:749812.

-40 Ikeda Y, Sato K, Pimentel DR, Sam F, Shaw RJ, Dyck JR, Walsh K: Cardiac-specific deletion of lkb1 leads to hypertrophy and dysfunction. J Biol Chem 2009;284:35839-35849. 